\title{
Efficacy of Siriraj, in-house-developed, frozen gloves for cold therapy reduction of chemotherapy-induced peripheral neuropathy in gynecological cancer patients: randomized controlled trial
}

\author{
Phreerakan Chitkumarn $^{1} \cdot$ Tharinee Rahong $^{1} \cdot$ Vuthinun Achariyapota $^{1}$ (D)
}

Received: 9 June 2021 / Accepted: 31 January 2022 / Published online: 11 February 2022

(c) The Author(s) 2022

\begin{abstract}
Objectives The primary objective of this study was to investigate the efficacy of cold therapy in reducing paclitaxel-based, chemotherapy-induced, peripheral neuropathy (CIPN). The secondary objective was to establish the incidence of CIPN arising from paclitaxel administration.

Materials and methods The study enrolled gynecological cancer patients who were aged over 18 years and receiving chemotherapy which included paclitaxel $\left(175 \mathrm{mg} / \mathrm{m}^{2}\right.$ every 3 weeks). The patients were allocated to control and cold-therapy groups by computer randomization. During paclitaxel administration, frozen gloves developed in-house by Siriraj Hospital were worn - with a cold pack inside — on both hands and both feet by the cold-therapy patients. The CIPN incidence was evaluated by FACT/GOG-Ntx (version 4) at each chemotherapy cycle and at the 1-month follow-up after treatment completion. Results There were 79 patients (control arm, 40; study arm, 39). The CIPN incidences in the control and cold-therapy groups were $100 \%$ and $48.7 \%$, respectively. CIPN was significantly decreased in the intervention group between the first cycle and the 1 month follow-up after chemotherapy cessation $(P$ value $<0.001)$. Four patients discontinued the cold therapy due to pain, but there were no serious adverse effects due to the therapy.

Conclusion The Siriraj Hospital, in-house-developed, frozen gloves can reduce CIPN effectively as part of cold therapy for paclitaxel-based chemotherapy. The benefits of using the gloves are apparent from the first chemotherapy cycle to the 1-month, post-treatment follow-up assessment.
\end{abstract}

Keywords Chemotherapy-induced peripheral neuropathy · Cold therapy · FACT/GOG-Ntx · Gynecological cancer · Cryotherapy $\cdot$ Paclitaxel

\section{Introduction}

The number of gynecological cancer patients is gradually increasing. The cancer type and its stage determine the treatment, for example, surgery, radiation, or chemotherapy. Taxanes, especially paclitaxel, are very commonly used for the chemotherapeutic treatment of gynecological cancers, such as ovarian cancer, cervical cancer, and endometrial cancer. However, paclitaxel has many frequent side effects,

Vuthinun Achariyapota

vuthinun.ach@mahidol.edu

1 Department of Obstetrics and Gynecology, Faculty of Medicine Siriraj Hospital, Mahidol University, Bangkok 10700, Thailand including bone marrow suppression, alopecia, myalgia, nausea and vomiting, and peripheral neuropathy.

Chemotherapy-induced peripheral neuropathy (CIPN) is one common side effect of taxanes. The incidence rate of CIPN varies from 20 to $100 \%$, and its severity depends on the cumulative dose of paclitaxel [1-3]. The symptoms can be either acute or chronic. Some patients develop acute symptoms after the first cycle of chemotherapy. Others, however, develop chronic symptoms after treatment completion, leading to disabilities and impaired quality of life [2]. CIPN affects the quality of life through the presence of pain and fatigue, impairment of cognitive function and mental health, and degradation of physical functioning [4]. Peripheral nerve damage can lead to either motor or sensory symptoms. The sensory symptoms are numbness, tingling, and burning pain, which eventually lead to motor symptoms [5]. Some symptoms may disappear 
after treatment discontinuation, but severe symptoms may persist for a prolonged period. The presence of CIPN necessitates discontinuation or dose reduction depending on severity, which consequently the treatment is not as effective as anticipated $[1,6]$.

Many studies have investigated the use of pharmaceutical and supplementary agents as well as alternative medicine for CIPN prevention and management. In Thailand, gabapentin and vitamin B complex are commonly prescribed for CIPN, despite the absence of a solid evidence base to support their use. In a study on ovarian cancer patients, gabapentin (900 mg a day) improved pain and neurological deficits in patients who developed peripheral neuropathy during carboplatin and paclitaxel administration. However, their quality of life did not change significantly [6]. The data are still inconsistent and limited; therefore, the American Society of Clinical Oncology clinical practice guidelines and review literature do not recommend CIPN treatment [7, 8]. In addition, a randomized, controlled trial examining whether vitamin B complex prevents CIPN found that the vitamin B complex could not significantly reduce CIPN more than a placebo [9]. Lastly, duloxetine was shown to significantly reduce CIPN in patients with gynecological cancer who received paclitaxel. However, the side effects of duloxetine were pronounced. They included somnolence, giddiness, nausea, constipation, dysgeusia, depression, and aggression, and they were particularly evident among elderly patients [10]. In addition, many trials studied acupuncture to reduce the incidence of CIPN. A recently published systematic review and meta-analysis concluded that although acupuncture was safe, symptom-alleviating effect on CIPN, however, could hardly be determined [11]. Like, electroencephalographic neurofeedback, yoga, therapeutic massage, their efficacy is still inconsistent. Further research is needed to understand their efficacy in preventing or treating the CIPN [4, 12].

Several studies have explored the effects of cold therapy, or "cryotherapy", on the prevention of CIPN. A previous study, experimental cooling of hands of healthy subjects to $19^{\circ} \mathrm{C}$ decreased blood flow to the palms about 53-60\% [13]. The hypothesis is that if the nerve blood flow is reduced during paclitaxel infusion, the level of paclitaxel at the periphery will be decreased accordingly. However, the effectiveness of cold therapy is still inconclusive. In previous prospective studies, cold therapy significantly lowered the incidence of CIPN arising from the usage of paclitaxel $[1,3,14]$. The current study investigated the efficacy of frozen gloves developed in-house by Siriraj Hospital. The gloves were used during cold therapy that was administered to prevent the development of CIPN in gynecological cancer patients receiving paclitaxel.

\section{Materials and methods}

\section{Patients}

This randomized, controlled trial enrolled gynecological cancer patients (ovarian, cervical, endometrial, and uterine cancers) who received chemotherapy that included paclitaxel. The drug was administered at a dosage of $175 \mathrm{mg} /$ $\mathrm{m}^{2}$ for $3 \mathrm{~h}$ every 3 weeks, concomitant with a platinumbased, or other, regimen. The study was conducted at the gynecological oncology ward of the Department of Obstetrics and Gynecology, Faculty of Medicine Siriraj Hospital, Mahidol University, Bangkok, Thailand, between May 2019 and November 2020. To be eligible for the intervention in this study, participants needed to be aged 18 years or older. Patients were not eligible if any subjective peripheral neuropathy symptoms were present before the chemotherapy initiation. They were also ineligible if they had potential peripheral neuropathy due to a prior taxane- or platinum-based chemotherapy regimen with disease recurrence within 1 year of that treatment's cessation. Also, excluded were participants who had rheumatoid arthritis, diabetes mellitus, or peripheral vascular disease, or who were using drugs to treat peripheral neuropathy symptoms. Written informed consent for participation in this study was obtained from all eligible patients with a new diagnosis and those with a cancer recurrence that had occurred more than 1 year after prior chemotherapy. The enrolled patients were randomized during the intervention period into 2 groups: "cold-therapy" and "control." Then their history chart was identified by a sticker to remind other medical staff not to prescribe any medication that would affect the CIPN. Patients in either group who discontinued chemotherapy early during the treatment ( $<4$ cycles) were excluded from the analysis. Before commencement of this research, its protocol was approved by the Siriraj Institutional Review Board (Si 267/2019). The trial was registered at the Thai Clinical Trials Registry (TCTR20210129001).

\section{Chemotherapy regimen}

The details of the 3 chemotherapy regimens used were as follows: paclitaxel $(175 \mathrm{mg} / \mathrm{m} 2)$ intravenous (IV) drip in $3 \mathrm{~h}$ plus carboplatin $(\mathrm{AUC}=5)$ IV drip in $1 \mathrm{~h}$; paclitaxel $(175 \mathrm{mg} / \mathrm{m} 2)$ IV drip in $3 \mathrm{~h}$ plus cisplatin $(50 \mathrm{mg} / \mathrm{m} 2)$ IV drip in $6 \mathrm{~h}$; paclitaxel $(135 \mathrm{mg} / \mathrm{m} 2$ on day 1$)$ IV drip in $3 \mathrm{~h}$ plus ifosfamide $(1600 \mathrm{mg} / \mathrm{m} 2$ on days $1-3)$ IV drip in $6 \mathrm{~h}$. 
Fig. 1 The Siriraj, in-housedeveloped, frozen gloves with 2 pieces of gel pack inside
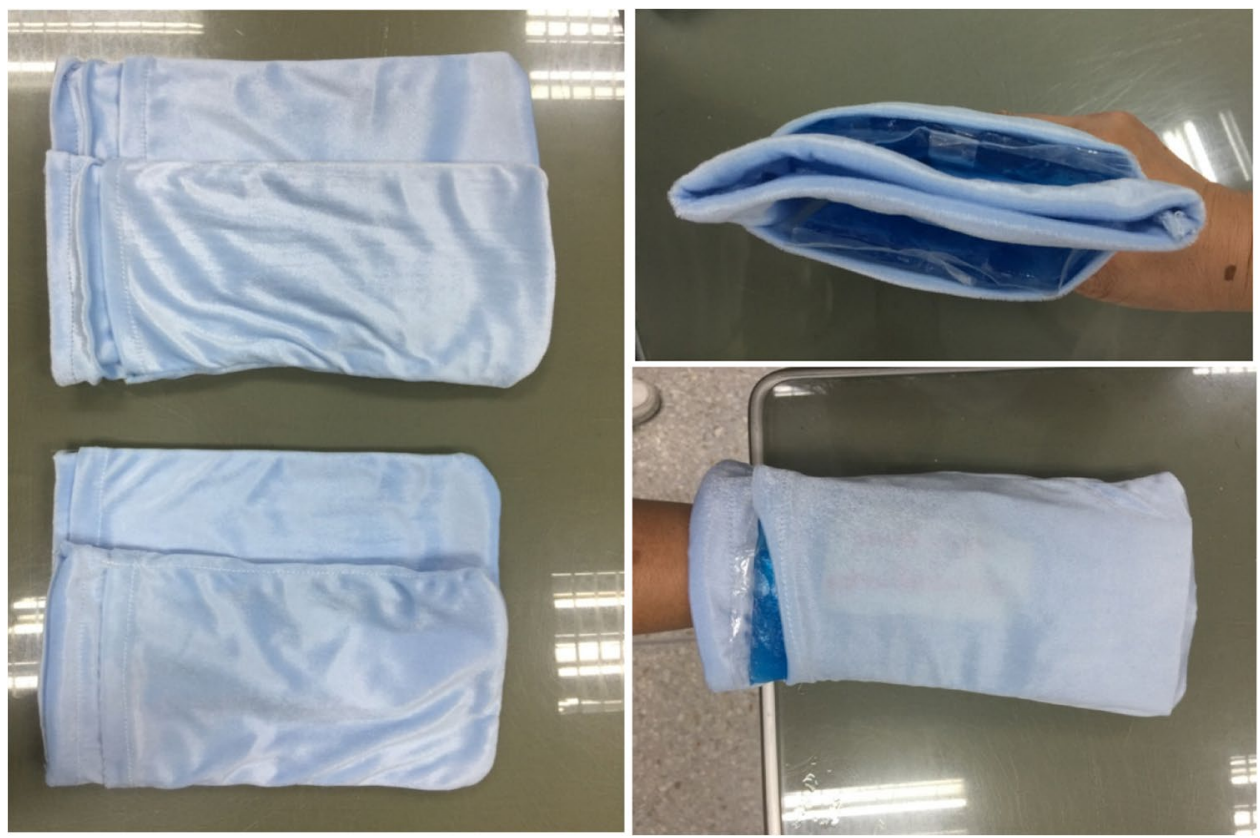

\section{Cold-therapy methodology}

The Siriraj, in-house, frozen gloves were developed using velvet fabric bags with 2 pieces of gel pack inside (Fig. 1). The gel pack (Nexcare; 3 M, St. Paul, MN, USA) was precooled in a refrigerator freezer before use. The velvet fabric bags were fitted over both hands and both feet for $3.5 \mathrm{~h}$. They were left in situ from $15 \mathrm{~min}$ before to $15 \mathrm{~min}$ after the paclitaxel was administered. The fabric bags were changed every $15 \mathrm{~min}$ to maintain the temperature at 10 to $20^{\circ} \mathrm{C}$ by the surface probe of digital hygro-thermometer.

\section{Evaluation criteria and timing}

The severity of CIPN was evaluated by questionnaire, using the Thai-version of the Functional Assessment of Cancer Therapy/Gynecologic Oncology Group-Neurotoxicity (FACT/GOG-Ntx; version 4). This health-related, quality-of-life questionnaire targets neurotoxicity-related chemotherapy treatment. It is comprised of 11 items, and patients report their sensory, motor, and auditory symptoms on a 5-point Likert-type scale ranging from 0 (not at all) to 4 (very much). The total score range is 0 to 44 , with a high score signifying less neurotoxicity. The clinically significant development of CIPN was defined as a decrease in the FACT/GOG-Ntx score by $>10 \%$ between 2 consecutive time points $[15,16]$. The evaluations for CIPN symptoms were conducted for up to 6 chemotherapy cycles, and a further evaluation was made at 1 month after the course finished.

\section{Primary and secondary objectives}

The primary objective was to determine the efficacy of the Siriraj, in-house-developed, frozen gloves when used during cold therapy to prevent the development of CIPN in gynecological cancer patients receiving paclitaxel. The secondary objective was to establish the incidence of CIPN resulting from paclitaxel administration.

\section{Sample size calculation}

According to a previous study, the incidences of CIPN in cold-therapy and control groups were $8 \%$ and $34 \%$, respectively [1]. Using an alpha of 0.05 and a power of $80 \%$ to detect differences between the 2 groups, a sample size of 38 cases was calculated for each group. After allowing for an estimated $20 \%$ dropout rate, 47 cases were set for each arm.

\section{Statistical analysis}

Statistical analyses were performed using IBM SPSS Statistics for Windows (version 27; IBM Corp., Armonk, NY, USA). The distribution of data was tested for normality using the Kolmogorov-Smirnov test. Descriptive data were summarized as mean ( \pm standard deviation $[S D]$ ), median (with interquartile range [IQR]), or frequency and percentage. Differences between the cold-therapy and control groups were determined by using the Student's $t$-test, Pearson's chi-squared test, or Wilcoxon Rank Sum test, as appropriate. Linear mixed modeling analysis was conducted to determine the differences in the mean FACT/GOG-Ntx 
scores of the cold-therapy and control groups over each chemotherapy cycle. $P$ values of less than 0.05 were deemed statistically significant.

\section{Results}

In all, 79 gynecological cancer patients received paclitaxelbased chemotherapy (40 in the control group, and 39 in the cold-therapy group). The consort flow of the participants is illustrated in Fig. 2. There were no statistically significant differences in the baseline data of the 2 groups (age, body mass index, body surface area, type of cancer, cumulative dose of paclitaxel and of platinum, total cycles of chemotherapy, and FACT/GOG-Ntx score; Table 1).

There was a statistical difference in the CIPN incidences of the 2 groups: $100 \%$ for the control group, versus $48.7 \%$ for the cold-therapy group $(P<0.001$; Table 2$)$. The difference was significant with the initial chemotherapy cycle: for the cold-therapy group, the incidence was $15.4 \%$ (6/39), while in the control group, it was $35 \%(14 / 40$; $P=0.045$; Table 3 ). As a result, the higher the number of chemotherapy cycles administered was, the greater the statistical difference between the CIPN incidences of the

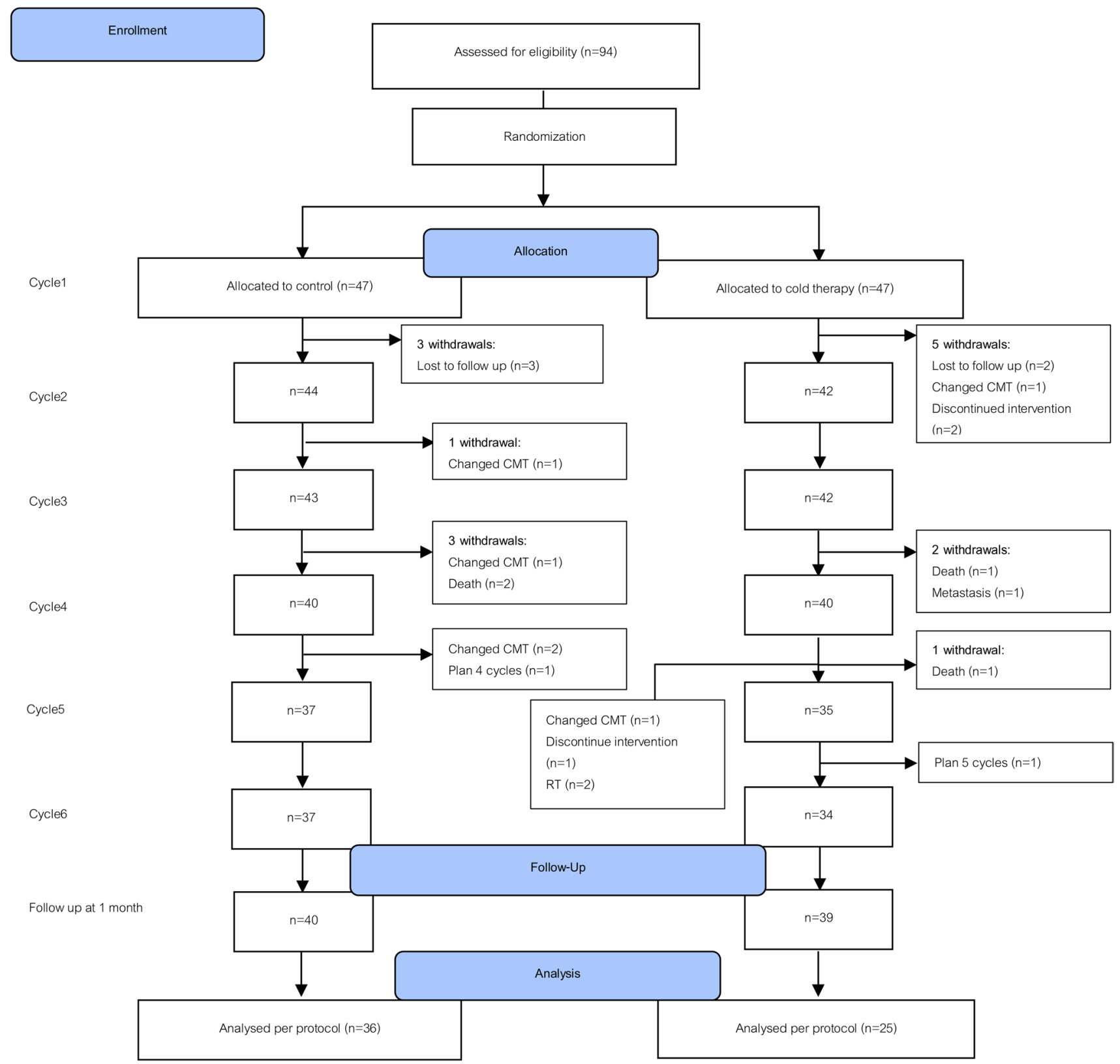

Fig. 2 Consort flow of the participant's diagram 
Table 1 Characteristics baseline data of participants

\begin{tabular}{llll}
\hline Characteristics & \multicolumn{2}{l}{ Mean \pm SD or $n(\%)$} & $p$ value \\
\cline { 2 - 4 } & $\begin{array}{l}\text { Control } \\
(n=40)\end{array}$ & $\begin{array}{l}\text { Cold therapy } \\
(n=39)\end{array}$ & \\
\hline Clinical & & & 0.441 \\
Age (year) & $55.45 \pm 13.21$ & $57.59 \pm 11.23$ & 0.205 \\
Body mass index $\left(\mathrm{kg} / \mathrm{m}^{2}\right)$ & $24.20 \pm 5.62$ & $22.76 \pm 4.29$ & 0.520 \\
Body surface area $\left(\mathrm{m}^{2}\right)$ & $1.55 \pm 0.20$ & $1.53 \pm 0.17$ & 0.283 \\
Cancer & & & \\
Ovary & $25(62.5 \%)$ & $21(53.8 \%)$ & \\
Endometrium & $11(27.5 \%)$ & $8(20.5 \%)$ & \\
Cervix & $3(7.5 \%)$ & $9(23.1 \%)$ & $1(2.6 \%)$ \\
Sarcoma & $1(2.5 \%)$ & & 0.553 \\
Platinum & & $4(10.3 \%)$ & \\
Cisplatin & $3(7.5 \%)$ & $34(87.2 \%)$ & \\
Carboplatin & $37(92.5 \%)$ & $1(2.6 \%)$ & 0.500 \\
Other (Ifosfamide) & $0(0 \%)$ & $1534.10 \pm 241.64$ & 0.978 \\
Cumulative dose of paclitaxel (mg) & $1569.00 \pm 215.08$ & $5.81 \pm 0.33$ & 0.580 \\
Total cycles per patient & $5.88 \pm 0.21$ & $43.44 \pm 1.27$ & \\
Baseline FACT score & $43.58 \pm 0.93$ & & \\
(Pre-chemotherapy) & & & \\
\hline
\end{tabular}

Data are mean $\pm \mathrm{SD}$, or number $(\%)$
Table 2 Incidence of CIPN in each group

\begin{tabular}{llll}
\hline CIPN & $\begin{array}{l}\text { Control } \\
(n=40)\end{array}$ & $\begin{array}{l}\text { Cold therapy } \\
(n=39)\end{array}$ & $p$ value \\
\hline Yes & $100 \%(40 / 40)$ & $48.7 \%(19 / 39)$ & $<0.001$ \\
No & $0 \%(0 / 40)$ & $51.3 \%(20 / 39)$ & \\
\hline
\end{tabular}

${ }^{*} C I P N=$ chemotherapy-induced peripheral neuropathy

2 groups became $(P<0.001)$. At the 1 -month follow-up session following the completion of the chemotherapy, the effectiveness of the cold therapy continued to be apparent. The CIPN incidence of the cold-therapy group (25.6\%) was significantly lower than that for the control group $(100 \% ; P<0.001$; Table 3).

The score for FACT/GOG-Ntx represented the severity of symptoms of CIPN and hence, the quality of life of the patients. After receiving the first chemotherapy cycle, the cold-therapy group's score was significantly better than that for the control group $(41.44 \pm 2.90$, versus $39.4 \pm 3.88 ; P=0.01)$. After completing the chemotherapy, at the 1-month review, the quality of life of the cold-therapy patients was still far superior to that of the control group $(40.64 \pm 5.70$, versus $30.13 \pm 5.13 ; P<0.001$; Table 3 and Fig. 3).

Four patients discontinued the cold therapy because they could not tolerate the discomfort caused by the low temperatures used. Apart from that, there were no substantial adverse effects, such as localized eczema or frostbite.

\section{Discussion}

CIPN is one of the troublesome, common, dose-limiting complications related to neurotoxic-chemotherapy agents, especially platinum and taxanes, commonly used with gynecological cancer patients. Taxane-based therapy can induce paresthesia, numbness, and pain in a stocking-andglove distribution (i.e., distal arms and legs) [17]. These symptoms affect the cancer-treatment process, necessitating discontinuation of the chemotherapy or, at least, a decrease in the paclitaxel dose. The severity of these symptoms depends on the cumulative dose of paclitaxel. Many pharmaceutical agents and supplements have been investigated to establish their efficacy in preventing or treating CIPN. Complementary and alternative medicines (for example, acupuncture) have been widely studied to explore their CIPN prevention and treatment potential. However, comprehensive scientific data in support of their use are still limited [18]. One alternative treatment modality, cold therapy (also termed cryotherapy), is a choice for CIPN management. It induces decreased blood flow to the target areas by vasoconstriction. In our recent investigation, we demonstrated a high incidence of CIPN as well as promising outcomes of our in-house-developed frozen gloves in lowering the CIPN rate.

In other studies, the incidence of CIPN varied from 20 to $100 \%$ of the patients who received paclitaxel [1-3]. In a comprehensive review, the author concluded that the rate was $68.1 \%$ when measured 1 month after the neurotoxicchemotherapy completion, $60 \%$ at 3 months, and $30 \%$ at 
Table 3 Score of FACT/GOGNtx questionnaire and incidence of CIPN in each cycle of chemotherapy between control and cold therapy groups
Fig. 3 Mean FACT/GOG-Ntx questionnaire score in each cycle of chemotherapy and at the follow-up period between control and cold therapy group

\begin{tabular}{|c|c|c|c|}
\hline & Control & Cold therapy & $p$ value \\
\hline \multicolumn{4}{|l|}{ Cycle 2} \\
\hline FACT score $($ mean $\pm \mathrm{SD})$ & $39.4 \pm 3.88$ & $41.44 \pm 2.90$ & 0.01 \\
\hline$\%$ Change of FACT score $($ mean \pm SD) & $-9.59 \pm 8.54$ & $-4.59 \pm 6.30$ & 0.004 \\
\hline$\%$ CIPN $(n)$ & $35 \%(14 / 40)$ & $15.4 \%(6 / 39)$ & 0.045 \\
\hline \multicolumn{4}{|l|}{ Cycle 3} \\
\hline FACT score $($ mean $\pm \mathrm{SD})$ & $37.92 \pm 3.44$ & $40.23 \pm 3.57$ & 0.005 \\
\hline$\%$ Change of FACT score $($ mean \pm SD) & $-12.94 \pm 7.89$ & $-7.28 \pm 8.92$ & 0.004 \\
\hline$\% \operatorname{CIPN}(n)$ & $57.5 \%(23 / 40)$ & $28.2 \%(11 / 39)$ & 0.009 \\
\hline \multicolumn{4}{|l|}{ Cycle 4} \\
\hline FACT score $($ mean $\pm \mathrm{SD})$ & $34.30 \pm 4.95$ & $40.87 \pm 4.73$ & $<0.001$ \\
\hline$\%$ Change of FACT score (mean \pm SD) & $-21.29 \pm 11.25$ & $-5.81 \pm 11.55$ & $<0.001$ \\
\hline$\% \operatorname{CIPN}(n)$ & $77.5 \%(31 / 40)$ & $17.9 \%(7 / 39)$ & $<0.001$ \\
\hline \multicolumn{4}{|l|}{ Cycle 5} \\
\hline FACT score $($ mean $\pm \mathrm{SD})$ & $34.03 \pm 5.43$ & $40.97 \pm 3.71$ & $<0.001$ \\
\hline$\%$ Change of FACT score (mean \pm SD) & $-21.85 \pm 12.39$ & $-5.55 \pm 9.92$ & $<0.001$ \\
\hline$\%$ CIPN $(n)$ & $89.2 \%(33 / 37)$ & $28.6 \%(10 / 35)$ & $<0.001$ \\
\hline \multicolumn{4}{|l|}{ Cycle 6} \\
\hline FACT score $($ mean \pm SD) & $31.05 \pm 5.75$ & $40.44 \pm 4.28$ & $<0.001$ \\
\hline$\%$ Change of FACT score (mean \pm SD) & $-28.69 \pm 13.04$ & $-6.92 \pm 11.11$ & $<0.001$ \\
\hline$\% \operatorname{CIPN}(n)$ & $94.6 \%(35 / 37)$ & $23.5 \%(8 / 34)$ & $<0.001$ \\
\hline \multicolumn{4}{|l|}{ Follow-up at 1 month } \\
\hline FACT score $($ mean $\pm \mathrm{SD})$ & $30.13 \pm 5.13$ & $40.64 \pm 5.70$ & $<0.001$ \\
\hline$\%$ Change of FACT score $($ mean \pm SD) & $-30.86 \pm 11.75$ & $-6.33 \pm 13.78$ & $<0.001$ \\
\hline$\% \operatorname{CIPN}(n)$ & $100 \%(40 / 40)$ & $25.6 \%(10 / 39)$ & $<0.001$ \\
\hline
\end{tabular}

Data are mean $\pm \mathrm{SD}$, or number $(\%), C I P N=$ chemotherapy-induced peripheral neuropathy

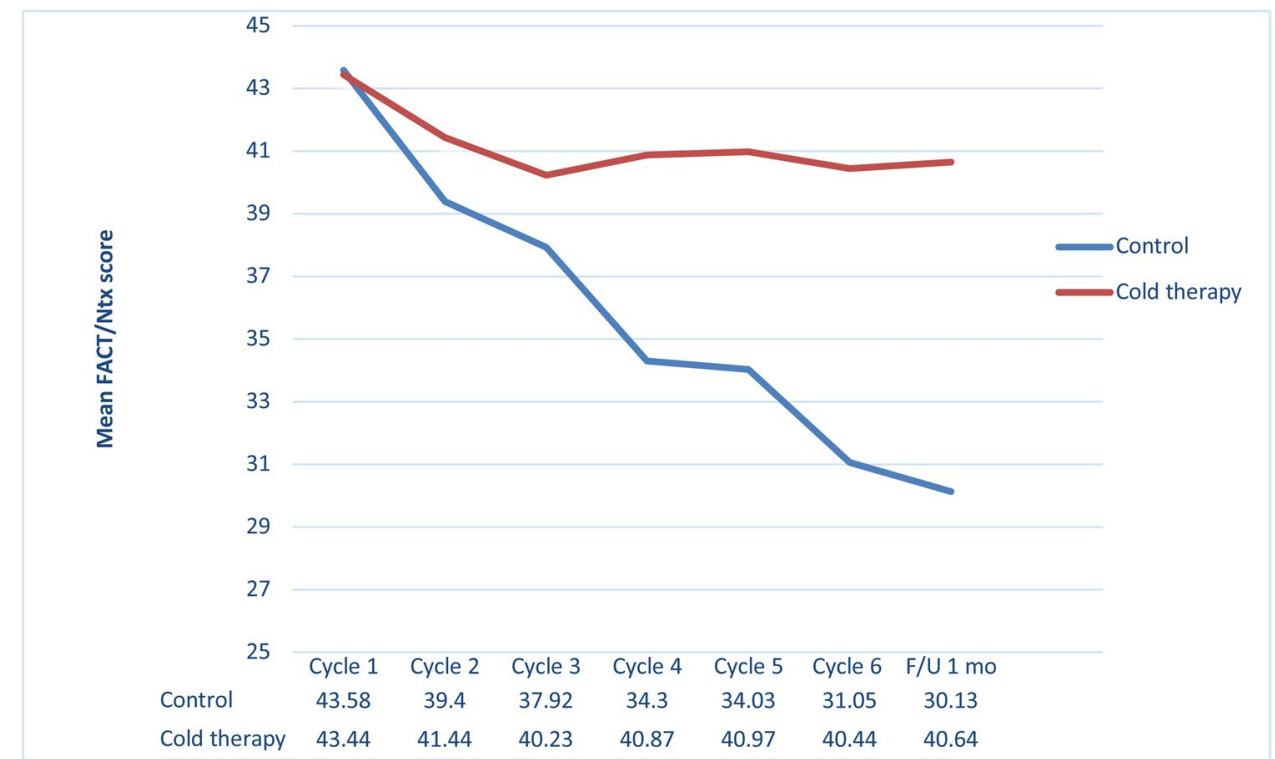

6 or more months [19]. In another recent work, the incidence was $100 \%$ for the control group and $48.7 \%$ for the cold-therapy group. The difference in the CIPN rates was striking from the completion of the first-cycle chemotherapy to the 3-month, post-treatment, follow-up session.
A similar finding was observed in the work of Sato et al. They investigated the use of cold therapy for gynecological cancer patients receiving tri-weekly, paclitaxel-based chemotherapy. Their results showed that there was a significantly lower incidence of peripheral neuropathy greater 
than grade 2 (under the Common Terminology Criteria for Adverse Events [CTCAE] grading system) for the cold therapy patients than for the controls [1]. Shigematsu and colleagues [14] demonstrated a reduction in the occurrence of CIPN when cold therapy was used for breast cancer patients receiving weekly paclitaxel. When assessed by the FACT/ GOG-Ntx questionnaire, the CIPN rate for the cold group was significantly lower than that for control group (41\% vs. $73 \% ; P=0.03)$. The incidence of peripheral neuropathy (either sensory or motor) $\geq 2$ under the CTCAE system was also significantly lower for the cold-therapy group. The outcomes of our study might be comparable to those of the earlier investigations because of our strict inclusion and exclusion criteria relating to the presence of peripheral neuropathy before receiving chemotherapy.

The key to success with cold therapy is achieving and maintaining the optimal surface temperature at the hands and feet. To ensure that vasoconstriction would decrease the blood flow to the hands and feet, we maintained the temperature between $10^{\circ} \mathrm{C}$ and $20^{\circ} \mathrm{C}$. A probe temperature was attached at the surfaces of the hands and feet to allow constant monitoring of their temperatures. However, some studies could not demonstrate the efficacy of cold therapy in reducing CIPN. In a multicenter, randomized, controlled trial, Beijers and coauthors investigated the efficacy of wearing commercial frozen gloves (Elasto-Gel Hypothermia TM-7006; Taureon, Nootdorp, Netherlands) to prevent CIPN during taxane treatment for breast and colorectal cancer. They concluded that there were no differences in the CIPN subscales of the cold-therapy and control arms [20]. It should be noted that the chemotherapy regimens employed in that study were heterogeneous. The taxanes used comprised oxaliplatin, paclitaxel, and docetaxel, with the majority of the participants $(60 \%)$ in each arm receiving oxaliplatin. Oxaliplatin-induced neurotoxicity symptoms include cold-intolerance, discomfort, and muscle cramps. Furthermore, $34 \%$ of the frozen gloves were discontinued before the end of treatment. Since this is a high dropout rate, the interpretation of the results might be prejudiced. Moreover, as that research team did not measure skin surface temperature, it may have been higher than the optimal level.

One strength of the present investigation was that, as it was a randomized controlled trial, allocation bias and confounding factors were minimized. However, some selection bias may still occur in the patients in the control arm or in the patients who declined to participate. In addition, the temperature of the iced-gloves was well controlled, so the efficacy of the iced-gloves was reliable. Moreover, the FACT/GOG-Ntx scoring system was a feasible, reliable, and validated instrument for the assessment of CIPN. It is also sensitive to meaningful clinical distinctions and changes over time [21]. Lastly, we developed an in-house frozen glove which is inexpensive, safe to use, and as effective as commercial frozen gloves in terms of preventing CIPN. At Siriraj, frozen gloves costs approximately $\$ 5$ per glove, a set of 4 gloves for both hands and both feet costs $\$ 20$. This compares very favorably with the typical charge of US $\$ 80$ for one commercial frozen glove, and $\$ 320$ for a set of 4 gloves. The temperature range of the Siriraj frozen gloves was $10^{\circ} \mathrm{C}$ to $20^{\circ} \mathrm{C}$, which was generally safe for the patients $[1,22]$. However, 4 patients stopped the cold therapy because they could not tolerate the discomfort caused by these temperatures. Otherwise, there were no major adverse effects, such as localized eczema or frostbite. A systematic review of 11 trials also suggested that there are no serious adverse events arising from cryotherapy [12].

One limitation of this study was the method of CIPN assessment. Although the FACT/GOG-Ntx questionnaire was validated, the questionnaire is a subjective report in that it depends on patients' experiences. Hence, it is less reliable than other assessment methods, such as the objective scores obtained with CTCAE (version 5) or the Total Neuropathy Score. The latter scoring system not only includes objective measures (such as pin prick, vibration threshold, and nerve conduction) but, importantly, a quality of life assessment [23]. The second limitation was the short follow-up period. CIPN can persist for a long time, so the follow-up period should be extended to 6 months to 1 year after chemotherapy. Another was that the current study was unblinded. Further study could put cold instead of ice gel packs in the control group to blind patients or health care providers to prevent bias. Finally, notwithstanding that cold therapy's efficacy is good, we did not evaluate the patients' satisfaction with using the frozen gloves. Hence, our future studies will use assessment tools that comprise subjective and objective evaluations, blinded studies, as well as long follow-up periods.

\section{Conclusion}

The Siriraj, in-house-developed, frozen gloves can reduce CIPN effectively as part of cold therapy for paclitaxel-based chemotherapy. The benefits are apparent from the first chemotherapy cycle to the 1-month, post-treatment follow-up assessment.

Acknowledgements The authors gratefully acknowledge Assist. Prof. Dr. Chulaluk Komoltri of the Division of Clinical Epidemiology, Department of Research and Development, Faculty of Medicine Siriraj Hospital, Mahidol University, for the assistance with the statistical analyses.

Authors' contributions Phreerakan Chitkumarn: conceptualization, investigation, data curation, writing - original draft, and final approval. Tharinee Rahong: conceptualization, investigation, validation, formal analysis, writing — original draft, and final approval. Vuthinun Achariyapota: conceptualization, design of the study, methodology, 
supervision, funding acquisition, writing — review and editing, and final approval. All authors read and approved the final manuscript.

Funding This study was funded by the Siriraj Research Development Fund, managed by Routine-to-Research (190G00035/023/19).

Data availability N/A.

Code availability N/A.

\section{Declarations}

Ethics approval The protocol was approved by the Siriraj Institutional Review Board (Si 267/2019). The trial was registered at the Thai Clinical Trials Registry (TCTR20210129001).

Consent of participate The participants have read and understood the provided information and given the written informed consent.

Consent for publication I, Vuthinun Achariyapota, hereby declare that I participated in the study and in the development of manuscript title, Efficacy of Siriraj, In-House-Developed, Frozen Gloves for Cold Therapy Reduction of Chemotherapy-Induced Peripheral Neuropathy in Gynecological Cancer Patients: Randomized Controlled Trial. I have read the final version and give my consent for the article to be published in Support Care in Cancer.

Conflicts of interest The authors declare that they have no conflicts of interest.

Open Access This article is licensed under a Creative Commons Attribution 4.0 International License, which permits use, sharing, adaptation, distribution and reproduction in any medium or format, as long as you give appropriate credit to the original author(s) and the source, provide a link to the Creative Commons licence, and indicate if changes were made. The images or other third party material in this article are included in the article's Creative Commons licence, unless indicated otherwise in a credit line to the material. If material is not included in the article's Creative Commons licence and your intended use is not permitted by statutory regulation or exceeds the permitted use, you will need to obtain permission directly from the copyright holder. To view a copy of this licence, visit http://creativecommons.org/licenses/by/4.0/.

\section{References}

1. Sato J, Mori M, Nihei S, Kumagai M, Takeuchi S, Kashiwaba M et al (2016) The effectiveness of regional cooling for paclitaxelinduced peripheral neuropathy. J Pharm Health Care Sci 2:33

2. Ibrahim EY, Ehrlich BE (2020) Prevention of chemotherapyinduced peripheral neuropathy: A review of recent findings. Crit Rev Oncol Hematol 145:102831

3. Hanai A, Ishiguro H, Sozu T, Tsuda M, Yano I, Nakagawa T et al (2018) Effects of cryotherapy on objective and subjective symptoms of paclitaxel-induced neuropathy: prospective self-controlled trial. J Natl Cancer Inst 110(2):141-148

4. Prinsloo S, Novy D, Driver L, Lyle R, Ramondetta L, Eng C et al (2017) Randomized controlled trial of neurofeedback on chemotherapy-induced peripheral neuropathy: A pilot study. Cancer 123(11):1989-1997

5. Song SJ, Min J, Suh SY, Jung SH, Hahn HJ, Im SA et al (2017) Incidence of taxane-induced peripheral neuropathy receiving treatment and prescription patterns in patients with breast cancer. Support Care Cancer 25(7):2241-2248

6. Magnowska M, Izycka N, Kapola-Czyz J, Romala A, Lorek J, Spaczynski M et al (2018) Effectiveness of gabapentin pharmacotherapy in chemotherapy-induced peripheral neuropathy. Ginekol Pol 89(4):200-204

7. Brewer JR, Morrison G, Dolan ME, Fleming GF (2016) Chemotherapy-induced peripheral neuropathy: Current status and progress. Gynecol Oncol 140(1):176-183

8. Hershman DL, Lacchetti C, Dworkin RH, Lavoie Smith EM, Bleeker J, Cavaletti G et al (2014) Prevention and management of chemotherapy-induced peripheral neuropathy in survivors of adult cancers: American Society of Clinical Oncology clinical practice guideline. J Clin Oncol 32(18):1941-1967

9. Schloss JM, Colosimo M, Airey C, Masci P, Linnane AW, Vitetta L (2017) A randomised, placebo-controlled trial assessing the efficacy of an oral B group vitamin in preventing the development of chemotherapy-induced peripheral neuropathy (CIPN). Support Care Cancer 25(1):195-204

10. Otake A, Yoshino K, Ueda Y, Sawada K, Mabuchi S, Kimura $\mathrm{T}$ et al (2015) Usefulness of duloxetine for Paclitaxel-induced peripheral neuropathy treatment in gynecological cancer patients. Anticancer Res 35(1):359-363

11. Hwang MS, Lee HY, Choi TY, Lee JH, Ko YS, Jo DC et al (2020) A systematic review and meta-analysis of the efficacy of acupuncture and electroacupuncture against chemotherapy-induced peripheral neuropathy. Medicine (Baltimore) 99(17):e19837

12. Samuels N, Ben-Arye E (2020) Integrative approaches to chemotherapy-induced peripheral neuropathy. Curr Oncol Rep 22(3):23

13. Nilsson AL (1987) Blood flow, temperature, and heat loss of skin exposed to local radiative and convective cooling. J Invest Dermatol 88(5):586-593

14. Shigematsu H, Hirata T, Nishina M, Yasui D, Ozaki S (2020) Cryotherapy for the prevention of weekly paclitaxel-induced peripheral adverse events in breast cancer patients. Support Care Cancer 28(10):5005-5011

15. Ajewole VB, Cox JE, Swan JT, Chikermane SG, Lamoth B, Iso $T$ et al (2020) Incidence of chemotherapy-induced peripheral neuropathy within 12 weeks of starting neurotoxic chemotherapy for multiple myeloma or lymphoma: a prospective, single-center, observational study. Support Care Cancer 28(4):1901-1912

16. Cella DF, Tulsky DS, Gray G, Sarafian B, Linn E, Bonomi A et al (1993) The Functional Assessment of Cancer Therapy scale: development and validation of the general measure. J Clin Oncol 11(3):570-579

17. Argyriou AA, Kyritsis AP, Makatsoris T, Kalofonos HP (2014) Chemotherapy-induced peripheral neuropathy in adults: a comprehensive update of the literature. Cancer Manag Res 6:135

18. Cheng XL, Liu HQ, Wang Q, Huo JG, Wang XN, Cao P (2015) Chemotherapy-induced peripheral neurotoxicity and complementary and alternative medicines: progress and perspective. Front Pharmacol 6:234

19. Seretny M, Currie GL, Sena ES, Ramnarine S, Grant R, MacLeod MR et al (2014) Incidence, prevalence, and predictors of chemotherapy-induced peripheral neuropathy: a systematic review and meta-analysis. Pain 155(12):2461-2470

20. Beijers AJM, Bonhof CS, Mols F, Ophorst J, de Vos-Geelen J, Jacobs EMG et al (2020) Multicenter randomized controlled trial to evaluate the efficacy and tolerability of frozen gloves for the prevention of chemotherapy-induced peripheral neuropathy. Ann Oncol 31(1):131-136

21. Huang HQ, Brady MF, Cella D, Fleming G (2007) Validation and reduction of FACT/GOG-Ntx subscale for platinum/paclitaxelinduced neurologic symptoms: a gynecologic oncology group study. Int J Gynecol Cancer 17(2):387-393 
22. Bandla A, Tan S, Kumarakulasinghe NB, Huang Y, Ang S, Magarajah $\mathrm{G}$ et al (2020) Safety and tolerability of cryocompression as a method of enhanced limb hypothermia to reduce taxane-induced peripheral neuropathy. Support Care Cancer 28(8):3691-3699

23. Curcio KR (2016) Instruments for assessing chemotherapyinduced peripheral neuropathy: a review of the literature. Clin J Oncol Nurs 20(2):144-151.
Publisher's note Springer Nature remains neutral with regard to jurisdictional claims in published maps and institutional affiliations. 